Revue d'histoire de l'Amérique française

REVUE D.HISTOIRE DE L'AMÉRIQUE FRANÇAISE

\title{
Deux procurations inédites de Jacques Cartier
}

\section{Robert Le Blant}

Volume 15, numéro 3, décembre 1961

URI : https://id.erudit.org/iderudit/302139ar

DOI : https://doi.org/10.7202/302139ar

Aller au sommaire du numéro

Éditeur(s)

Institut d'histoire de l'Amérique française

ISSN

0035-2357 (imprimé)

1492-1383 (numérique)

Découvrir la revue

Citer ce document

Le Blant, R. (1961). Deux procurations inédites de Jacques Cartier. Revue

d'histoire de l'Amérique française, 15(3), 443-449.

https://doi.org/10.7202/302139ar d'utilisation que vous pouvez consulter en ligne.

https://apropos.erudit.org/fr/usagers/politique-dutilisation/ 


\section{DEUX PROCURATIONS INÉDITES DE JACQUES CARTIER}

\section{9 janvier 1541}

Deux procurations de Jacques Cartier découvertes par $\mathbf{M}^{\mathrm{r}}$ Jean Sterlin, membre de la Société d'Histoire de France, qui nous les a fort aimablement communiquées, ont été déchiffrées avec l'aide précieuse de $\mathrm{M}^{\mathrm{me}}$ Jurgens, conservateur au Minutier central des Archives Nationales. Figurant au magnifique registre des minutes de Jean Boreau pour le début de l'année 1540, soit 1541 d'après la computation actuelle, puisque l'année commençait à Pâques lors de la rédaction, elles ne sont malheureusement pas signées. Cette omission qu'on rencontre fréquemment dans les minutes des notaires parisiens du milieu du XVI siècle ne peut inspirer aucune déduction sur l'habileté très éventuelle du découvreur dans le maniement de la plume, ${ }^{1}$ mais les textes apportent des précisions sur son activité et certains de ses probables compagnons. Les deux actes passés le même jour se complètent. Ils ont pour objet de constituer des procureurs ayant mission de se faire remettre des prisonniers, sauf un, devant être conduits à leurs frais à Saint-Malo pour participer au troisième voyage de Cartier. Celui dont la contribution pécuniaire n'est pas prévue est gratifié d'une surveillance sérieuse.

Les procureurs furent, dans le premier acte, un avocat au Grand Conseil, Jean de Montescot, dans le second, Jacquet et Mathurin Blancvillain, nommés sans indication complémentaire pouvant faciliter une identification plus complète. Les deux procurations commencent par une analyse des lettres patentes du 17 octobre 1540 qui avaient autorisé le Malouin à enlever 50 prisonniers. ${ }^{2}$ C'est en application de cette faveur que Cartier, occupé tant à recruter des gens de mer qu'à se pourvoir de bonnes munitions et autres choses nécessaires, eut recours à des mandataires pour constituer une partie de son équipage évidemment formé de personnages quelque peu sédentaires.

Il apparaissait, d'après le texte des lettres du 17 octobre 1540, que le navigateur pouvait choisir toutes personnes détenues, à l'exception des coupables de certains crimes. Cependant le savant et très érudit Marichal, au cours de ses fructueuses recherches sur l'activité de Roberval devant les parlements, ${ }^{3}$ a

\footnotetext{
1 RHAF, XV (juin 1961).

2
3
3 Humanisme et renaissance I $(1934), 8^{\circ} \mathrm{Z} 26830$, à la Bibliothèque Nationale.
} 
été d'avis que les facultés données à Cartier ne s'étendaient pas aux ressortissants de ces juridictions. Sans doute, le mandement du 17 octobre 1540 spécifiait seulement les prévôts, baillis et sénéchaux, tandis que les lettres des 7 février et 9 mars 1541 accordées à Roberval précisaient être adressées « à nos amés et féaux conseillers, les gens tenant nos cours de parlement de Paris, Toulouse, etc.,$^{4}$ mais la portée de l'acte du 17 octobre 1540 pouvait être considérée comme générale sur la compétence par l'adjonction « et aultres nos justiciers et officiers tant de nostre dict royaume que de nostre pays de Bretagne ». On retrouve une formule analogue dans les lettres données par le Dauphin, le 20 octobre 1540 , « et tous nos aultres justiciers.$^{5}$ La question se trouve maintenant résolue par l'analyse donnée aux procurations spécifiant «toutes les prisons de ce royaume 》 et le mandat de la première procuration encore plus précis: "de se transporter par devers toutes les cours et juges ». Un prisonnier dépendant du parlement de Bordeaux avait du reste été déjà réclamé. Cette première procuration au profit de Jean de montescot est le document le plus long, ayant une portée géographique étendue. Le bénéficiaire est chargé de se transporter dans toute la France pour prendre 12 prisonniers en plus des 8 pour lesquels mandat lui avait été donné dès le dernier jour d'octobre 1540, afin de parfaire un nombre de 20 . Un certain détail du mandat précédent est donné, car il s'agissait de 6 détenus à Paris et de 2 à Chartres qui semblent avoir été enlevés, mais on ne sait à quelle juridiction.

Les premières instructions du découvreur avaient été au moins en partie orales, car il ratifia le choix fait pour être mis à sa disposition quand il les irait quérir de Germain de Pierrebussière et de deux de ses complices prisonniers à la conciergerie du parlement de Bordeaux, personnages paraissant devoir être compris dans les douze faisant l'objet de la procuration écrite.

Nous avons pu trouver quelques renseignements complémentaires sur Jean de Montescot, avocat au Grand Conseil et maître des requêtes de la reine. Il était le fils de Pierre I ${ }^{\text {er }}$ de Montescot, avocat à Chartres, et de Marie de Givès, fille de Renaud de Givès, seigneur de Baronville, avocat, prévôt de Chartres, et d'Anne Cadot. Sa mère se remaria avec Jacques Blanchard, ayant eu deux enfants de son premier mariage: Pierre II et Marthe. Pierre II de Montescot se maria avec Marguerite

4 Biggar, op. cit.

5 Idem. 
Blanchard, fille de Jacques, et Marthe de Montescot épousa Martin Veillard, receveur des décimes à Verneuil. ${ }^{6}$ Jean de Montescot se maria, lui-même, avec Nicole de Mareau dont il eut Claude de Montescot, seigneur de Baconville, receveur des parties casuelles, promu secrétaire du roi, le 3 août 1571, qui eut plusieurs enfants de son mariage avec Madeleine Averdin. ${ }^{7}$

Quant à Germain de Pierrebussière, les représentants de sa famille, portant de sable au lion d'or, ${ }^{8}$ sont habituellement, dans les dossiers généalogiques, qualifiés de Pierrebuffière, nom d'un chef lieu de canton de la Haute-Vienne, arrondissement de Limoges, situé entre cette ville et Uzerche. Leur véritable nom peut avoir été de Châteauneuf, d'après l'acte de création de deux foires au village de Chabanes en Limousin, de juillet 1542, dans lequel intervint un Louis de Châteauneuf, dit de Pierrebuffière. $^{9}$ Les de Pierrebuffière prétendaient tirer de la baronnie de ce nom le titre de premier baron du Limousin. Germain était le troisième fils de Louis $\mathrm{I}^{\text {er }}$ de Pierrebuffière et de Marguerite de la Roche-Aymond. Son père était qualifié vicomte de Combord, seigneur baron de Chasteauneuf de Peyrat et de Freignet, seigneur de Chabanes, Beaumont et Chambaret. Son frère, François de Pierrebuffière, aussi vicomte de Combor, baron de Chasteauneuf, épousa Catherine de Ségur, fille de Gaston de Ségur et de Jeanne de Chassagne, ${ }^{10}$ puis Jeanne Chabot, fille de Charles Chabot, seigneur de Sainte-Foy et de Madeleine de Puyguyon. Son autre frère, Louis II, sieur de Chabanes, épousa Isabeau de Ségur, tandis que Germain épousa Progente de Lais. Les successeurs semblent bien avoir porté des noms de seigneuries suivis des titres conférés par elles, car messire Charles de Pierrebussière se qualifia «du Pierrebussière et de Combord, vicomte de Combord, baron de Pierrebussière $\gg .11$ Une branche peut avoir subsisté en Berry et à Paris jusqu'à la fin de l'ancien régime. ${ }^{12}$

Quant à la mission de Jacquet et Mathurin Blancvillain, elle était assez restreinte, puisqu'ils devaient seulement se rendre à Chartres pour réclamer au prévôt de cette ville, prendre et conduire ou faire conduire sous bonne garde le prisonnier Michel

6 Dossiers Bleus 458, art. Montescot, à la Bib. Nationale.

7 Pièces Originales 2015, art. Montescot, idem.

8 Dossiers Bleus 523, art. Pierrebuffière, idem.

9 Catalogue des actes de François Ier, IV: 335 , acte 12670 , à la disposition des lecteurs de la salle de travail des manuscrits, idem.

10 Dossiers Bleus 609, art. Ségur, idem.

11 Minutes de Coutenot, XVI: 91, aux Archives Nationales. fière.

12 La Chesnaie-Desbois, Dictionnaire de la Noblesse, art. Pierre Buf- 
Blanchard à Saint-Malo pour y être embarqué avec les autres dont Cartier avait la charge. La similitude du nom de cet élu avec celui de certains alliés de Jean de Montescot peut expliquer la procuration spéciale consentie aux Blancvillain.

ROBERT LE BLANT

\section{PIEECES JUSTIFICATIVES}

Fut présent en sa personne noble homme Jacques Cartier, capitaine général et maistre pillote de l'armée et expedition ordonnée par le Roy nostredict seigneur pour aller au voiage des terres de Canada, archillagua et Sacquenay disant que pour autant que par la commission et pouvoir à lui donné par le Roy nostredict seigneur par ces lettres patentes données à Sainct Pris le dixseptiesme jour d'octobre dernier passé il luy est permis et donné puissance prendre par toutes les prisons de ce Royaulme le nombre de cinquante prisonniers prévenus et accusés de quelzconques cas et crimes excepté crimes de leze magesté, hérezie et faulce monnoye et iceulx prisonniers choisir et eslire ou faire choisir ou eslire par ses commis et depputes pour iceulx prisonniers mener audict voiaige en mandant par le roy nostredict seigneur à tous juges delivrer lesdicts prisonniers jusques audict nombre a icelluy Cartier ou sesdicts commis selon le choix qu'ils en feront et que icelluy Cartier ne peult a present vacquer à aller par toutes les prisons de ce royaulme pour choisir lesdicts prisonniers, ains luy est nécessaire dresser son equippaige marin et se pourveoir de bonnes munitions et aultres choses nécessaires a faire ledict voiaige, pour ces causes et considérations et afin que le dict voiaige ne soit retardé et que ledict nombre desdicts prisonniers puisse estre choisi et levé pour mener audict voiaige, icelluy Cartier, de son bon gré, pure et libere (sic) volunté recongnut et confessa en la presente par devant lesdicts notaires comme par devant nous en droict jugement avoir commis etc par la teneur ${ }^{13}$ ces presentes commect comme son lieutenant et commis noble homme et sage maistre Jehan de Montescot advocat au grant conseil du roy nostredict seigneur a ce present auquel il a donné et donne en vertu du pouvoir a luy donné par le roy nostredict seigneur plain pouvoir, puissance, auctorité et mandement especial de soy transporter en toutes ${ }^{14}$ de ce royaulme et par devers toutes les cours et

13 Les mots «de cesdictes» qui figurent dans la procuration donnée à Jacquet et Mathurin Blancvillain ont été omis.

14 Deux mots effacés par l'humidité. 
juges ${ }^{15}$ pour esdicts lieux, comme son commis, choisir, eslire, prandre des prisonniers de la qualité permise, prandre par lesdictes lettres patentes du roy jusques au nombre de douze oultre par dessus le nombre de huict qu'il luy a par ci devant dés le penultieme jour d'octobre dernier passé donné puissance choisir et prendre six és prisons de ceste ville de Paris et deux és prisons de la ville de Chartres, lesquelz huict avec lesdicts douze font le nombre de vingt prisonniers qu'il veult et permet audict de Montescot choisir et prandre partout esdict royaulme et a ceste fin qu'il puisse comme son commis bailler toutes requestes et faire toutes poursuictes au cas nécessaire pour la delivrance dudict nombre de vingt prisonniers et a ceste fin qu'il puisse substituer en son lieu et absence ung ou plusieurs procureurs ayans tel pouvoir que luy de laquelle delivrance faicte audict de Montescot, ledict Cartier dés a present, comme dés lors, s'est chargé et charge et promect soubz l'obligation de tous ses biens l'en descharger envers le roy et tous juges et personnes comme si la delivrance desdicts prisonniers luy estoient personnellement faicte pour lesdicts prisonniers ainsi délivrés mener ou faire mener par ledict de Montescot en la ville de Sainct Mallo en Bretaigne ou ledict Cartier entend et ordonne qu'ils soient menez aux despens des dicts prisonniers pour illec les enbarquer avec luy et si aucuns par ledict de Montescot desjà choisis ou a choisir luy estoient reffusez, veult et entend qu'il en puisse choisir en leur lieu aultres jusques audict nombre de vingt seullement et generallement et sepeciallement par cesdictes presentes quant a prendre et choisir le nombre de ses vingt prisonniers par toutes les prisons de cedict royaulme, icelluy Cartier a commis et commect icelluy de Montescot et luy a donné et donne tel pareil pouvoir, puissance et auctorité que le roy luy a donné par cesdictes lettres patentes, promectant icelluy Cartier avoir pour aggreable, ferme et estable tout ce que par ledict de Montescot sera faict et besongné en ce que dict est, circonstances et deppendances et de ce descharger et desdommager icelluy de Montescot, declarant oultre qu'il a pour aggreable le choix que ledict de Montescot a faict de Germain de Pierrebussiére et deux aultres de ses complices estans prisonniers és prisons de la conciergerie de la court de parlement de Bourdeaulx pour quant icelluy Cartier les yroit querir luy estre delivrés, ce que ledict de Montescot a faict pour arrester lesdicts de Pierrebussiere et complices seullement soubs le vouloir d'icelluy Cartier dont duquel choix ains faict par ledict de Montescot et de ce que a escript a ladicte court pour cest effect, icelluy Cartier l'a deschargé et descharge comme faict

15 Un mot, idem. 
par son adveu et mandement verbal. Promect oultre ledict Cartier par les foy et serment de son corps és mains desdicts notaires le contenu cy dessus avoir et tenir pour bien aggreable, ferme et estable a tousiours sans jamais a nul jour par luy ne par aultre aucunement y contrevenir soubz l'obligation de tous et chacuns ses biens et de ceulx de ses hoirs, meubles, immeubles presents et advenir pour ce par luy soubzmis a la juridiction et contraincte de ladicte prévosté de Paris et de toutes aultres justices et juridictions ou trouvez seront et renoncer en ce faisant expressement par cesdicts sermens et foy à toutes choses a ce contraires et au droict disant generallement renonceans non valloir. Faict l'an mil cinq cens quarente le dimanche neufiesme jour du moys de janvier.

Claude Boreau Jean Boreau. ${ }^{16}$

Fut present en sa personne noble homme Jacques Cartier, cappitaine général et maistre pillote de l'armée et expedition ordonnée par le Roy nostredict seigneur pour aller au voiage des terres de Canada, archillagua et Sacquenay, disant que pour autant que par la commission et pouvoir a lui donné par le Roy nostredict seigneur par ces lettres patentes données à Sainct Pris le dixseptiesme jour d'octobre dernier passé il luy est permis et donné puissance prendre par toutes les prisons de ce royaulme le nombre de cinquante prisonniers prévenuz et accusez de quelconquez cas et crimes excepté crimes de leze magesté, herezie et faulce monnoye et tous prisonniers choisir et eslire ou faire choisir et eslire par ses commis et depputez pour iceulx prisonniers mener audit voiage en mandant par le Roy nostredict seigneur a tous juges de livrer lesdicts prisonniers jusques audict nombre audict Cartier ou sesdicts commis selon le choix qu'ils en feront et que icelluy Cartier ne peult a present vacquer a aller choisir par toutes les prisons de ce royaulme lesdicts prisonniers, ains luy est necessaire dresser son equippage marin et se pourveoir de vivres, munitions et aultres choses nécessaires afaire ledict voiage, pour ces causes et considérations et affin que ledit voiage ne soit retardé et que ledict nombre desdicts prisonniers puisse estre choisy et levé pour mener audict voiage, icelluy Cartier, de son bon gré, pure et libere (sic) volunté recongnut et confessa en la presente et par devant lesdictz notaires comme par devant nous en droict jugement avoir commis et par la teneur

16 Archives Nationales, Minutier central, VIII : 60, fo. 241. 
de cesdictes presentes commect en son lieu et comme ses lieutenans lesdicts Jacquect et Mathurin Blancvillain pour et en son lieu et comme sesdicts commis par eulx ou l'un d'eulx en l'absence de l'aultre aller et eulx transporter en la ville de Chartres par devers les bailly et prevost dudict lieu ou leurs lieutenans et illec demander, choisir et prandre pour mener audict voiaige Michel Blanchard prisonnier es prisons de Chartres seullement pour icelluy mener ou faire mener et conduyre soubz bonne et sure garde a Sainct Mallo en Bretaigne pour illec estre enbarqué et mené avec les aultres dont ledict Cartier a la charge, promectan oultre ledict Cartier par les foy et serment de son corps es mains desdicts notaires le contenu cy dessus avoir et tenir pour bien aggreable, ferme et estable a tousiours sans jamais à nul jour par luy ne par aultres y contrevenir soubs l'obligation de tous et chacuns ses biens et de ceulx de ses hoirs meubles et immeubles presens et advenir pour ce par luy soubmis à la juridiction et contraincte de ladicte prévosté de Paris et de toutes aultres justices et juridictions ou trouvez seront et renoncer en ce faisant expressement par sesdicts serment et foy a toutes choses a ce contraires et au droict, disant generallement renonceans non valloir. Faict, l'an mil cinq cens quarente, le dimanche neufiesme jour du moys de janvier.

Claude Boreau Jean Boreau. ${ }^{17}$

17 Archives Nationales, Minutier central, VIII : 60, fo. 243. 